\title{
Differential Gene Expression and Transcriptomic Analysis of ATG8 and ATG5 by Engineered Accumulation of Polyamines during Tomato Fruit Ripening
}

\author{
Mohamed Ibrahim* \\ Department of Botany, Faculty of Science, Ain Shams University, 11566 Cairo, Egypt
}

\section{A R T I C L E I N F O}

Article history:

Received 01 August 2017

Accepted 20 August 2017

Keywords:

Solanum lycopersicum L.;

Fruit ripening;

Polyamines;

S-Adenosyl Methionine;

Decarboxylase (SAMdc);

Autophagy process.

\begin{abstract}
A B S T R A C T
Autophagy process plays a pivotal role in nutrient recycling which maintains cellular homeostasis during growth and developmental stages in many eukaryotes. Autophagy-related proteins 5 and 8 (ATG5 and ATG8) are responsible of playing an indispensable role in formation of mature autophagosome that fuses with vacuole to transfer cellular cargo for digestion and recycling. On the other hand, polyamines (PAs) are ubiquitous polycationic amines that contribute in numerous biological processes and are involved in regulation at both transcriptional and translational levels. To figure out whether PAs play a role in the ripening-associated autophagy process, transcriptomic analysis of ATG8/ATG5 genes and analysis of their protein products were performed. RNA sequencing (RNASeq) experiment was executed using Solanum lycopersicum L. cv. Ohio 8245 (WT) and its transgenic homozygous (HO) lines (556HO and 579HO) expressing yeast S-Adenosyl Methionine Decarboxylase $(y S A M d c)$ under SIE8, a fruit-ripening promoter. Normalization of read counts of differentially expressed genes was performed. Gene transcripts of nine putative autophagy genes of both ATG8 and ATG5 were identified (two for ATG5 and seven for ATG8). It was found that gene expression of ATG8/ATG5 gene transcripts were altered during fruit ripening in PAs enriched genotypes. In addition, to decipher whether gene expression behavior is maintained on the protein level, total protein extraction, SDS-PAGE, and immunoblotting analyses were carried out. ATG8/ATG5-conjugates were found to be differentially and specifically expressed in $556 \mathrm{HO}$ and/or $579 \mathrm{HO}$ genotypes. Presented study has provided evidences that $y S A M d c$-high spermidine and spermine play a role during fruit ripening by altering the steady state levels of ATG8/ATG5. Therefore, it might be speculated that triggered alteration in autophagy-related genes (ATG8/ATG5) could be regarded as one mechanistic key pathway of PAs to maintain cellular homeostasis, increase longevity, development, and ripening of tomato fruit.
\end{abstract}

\footnotetext{
Introduction

All organisms have evolved through recycled pathways for their intracellular constituents as an essential way for the optimal growth and developmental processes ${ }^{[1-3]}$. Autophagy machinery is considered as recycling pathway in which the surplus organelles or the protoplasmic aggregates (cargo) are encapsulated by autophagosomes ${ }^{[3-5]}$. Autophagy process plays a significant role in nutrient recycling/mobilization/reassimilation during seed germination and seedling establishment ${ }^{[\mathbf{6 - 9}, \mathbf{1 2}]}$, controlling seasonal senescence ${ }^{[7]}$,

catabolizing various metabolites or protoplasmic components, and has also a housekeeping role in removing the cellular ${ }^{[10-11]}$. Under nutrient deprivation, autophagy provides adequate supply of alternative energy sources like amino acids that can be transformed into intermediates of the Krebs cycle producing energy for the production of ATP molecules ${ }^{[13]}$. In addition, autophagy is implicated in pathogen avoidance and resistance mechanisms ${ }^{[14-16]}$ by enhancing the hypersensitivity response which is sort of the Programmed Cell Death (PCD) in the infected area that leads to necrosis. Autophagy also has a vital role in chloroplast maturation and repair ${ }^{[17]}$. In the absence of 
autophagy regulated recycling process, cells would accumulate undesirable cargo with its constituents causing a general toxicity and growth inhibition for the living organism ${ }^{[\mathbf{1 8 - 2 3}]}$. Moreover, autophagy is highly inducible with stresses or even sustained at a basal level under certain physiological conditions ${ }^{[24-26]}$. Multiple proteins are responsible for the formation of autophagosome, the subcellular complex responsible for autophagy process, especially Autophagy-Related Protein 5 (ATG5) and ATG8 (Figure 1). Generally, five core autophagy complexes are required for autophagy events including, ATG1/ATG13 kinase complex, ATG9 complex, phosphoinositide 3-kinases (PI3K) Complex, ubiquitin-like ATG8-phosphatidyl ethanolamine (PE) conjugation, and finally ubiquitin-like ATG12-and ATG5 conjugation pathway ${ }^{[27-30]}$. The genes involved in the autophagy process were firstly identified in Saccharomyces cerevisiae based on absence or reduction of autophagosome in nutrient starved cultures $[5,31-33]$. More than 30 genes have been identified as ATG genes. The homologues of ATGs were recognized in many organisms including animals and plants revealing the highly conserved autophagic pathway in eukaryotic life ${ }^{[28,34-35]}$. The role of autophagy was characterized and functionally analyzed in different plant biological systems. In Arabidopsis thaliana L. mutations in some of autophagy-related genes have exhibited a number of phenotypes. However, autophagic machinery genes in other plants, such as Zea maize ${ }^{\text {[36- }}$ ${ }^{37]}$, Oryza sativa ${ }^{[38-39]}$, Triticum ${ }^{[40]}$, and Solanum lycopersicum ${ }^{[\mathbf{4 1 ]}}$ need to be further investigated.
Many phyto-hormones, especially ethylene, play essential roles in fruit development and ripening ${ }^{[42-44]}$. Polyamines (PAs) have been shown to extend shelf life and reported to increase longevity in several organisms, including tomato fruits ${ }^{[45-47]}$. Biogenic amines, putrescine, spermidine and spermine impact plant senescence, decay symptoms, and fruit ripening in Solanum lycopersicum [47]. PAs are ubiquitous polycationic amines that contribute in numerous biological processes such as growth, development, and senescence, and involved in regulation at both transcriptional and translational levels ${ }^{[48-55]}$. PAs have essential roles in cell proliferation and differentiation ${ }^{[\mathbf{5 4},}$ 56-58]. In many organisms, PAs are considered as juvenility factors and are associated with cell deathrelated processes ${ }^{[59-60]}$. However, the molecular mechanisms regulating effects of PAs in plants are not yet understood. Understanding these mechanisms would likely provide a method to improve yield and shelf life of fresh product. Additionally, transcriptomic, proteomic and metabolomics investigations have shown that fruit undergo massive physiological changes during maturation and seed dispersal ${ }^{\text {[61] }}$. Once reach maturity (maximal size) tomato fruit can ripen both on-planta and off-planta. Under both conditions, especially off-planta ripening, fruit has to mobilize resources within fruit, a process that likely requires autophagy. Role of autophagy process during the ripening of fleshy fruit has not been investigated yet. Shelf life of fruit is normally determined by the rate of change of this metabolic activity leading to fruit softening. This process that last

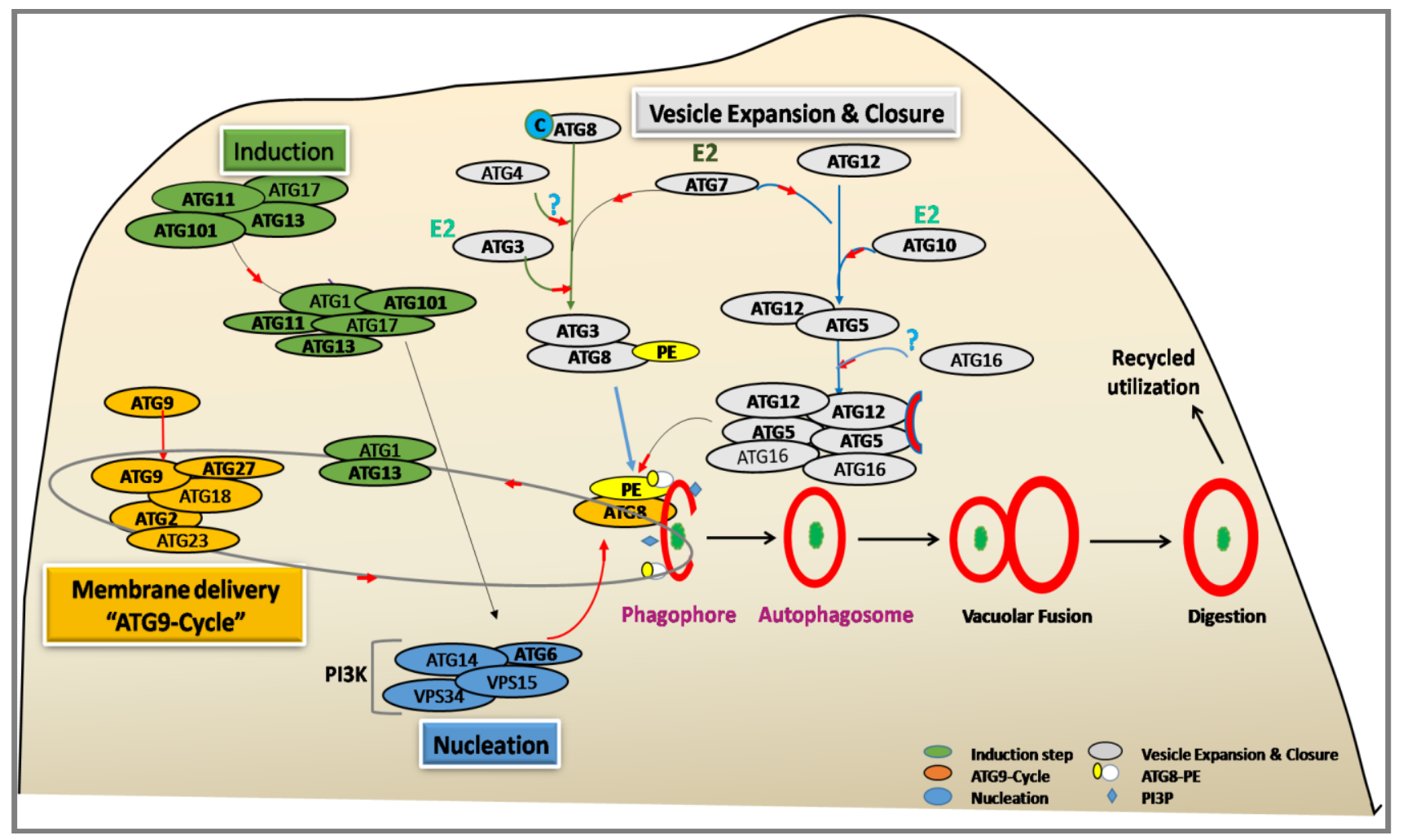

Fig. 1: Tentative schematic representation of known or proposed steps within the autophagy (ATG) pathway in plant. ATG proteins that have been expressed in tomato fruits are highlighted in orange color. The other genes expressed in other plants such as Arabidopsis are highlighted in blue-gray color. 
several days to weeks require reallocation of resources to provide energy source and build blocks for new protein synthesis, especially those regulating catabolism of cell wall material ${ }^{[62]}$. Earlier investigations have shown the advantage of PAs rich tomato homozygous independent genotypes $(556 \mathrm{HO}$ and $579 \mathrm{HO})$ in slow aging ${ }^{[45]}$. To figure out whether PAs play a role in the ripening-associated autophagy process, transcriptomic analysis of ATG8/ATG5 genes that was consequently supported with the analysis of their protein products was performed.

\section{Materials and Methods}

\section{Plant Material and fruit collections}

Solanum lycopersicum L. cv. Ohio 8245 (WT), and its transgenic homozygous (HO) lines $(556 \mathrm{HO}$ and $579 \mathrm{HO})$ expressing yeast SAMdc gene $(y S A M d c)$ under SIE8, a fruit-ripening promoter ${ }^{[45]}$, were used in this study. Tomato plants were grown in greenhouse facility at Horticulture and Landscape Department, Purdue University under normal practices. Wild type plants (WTs) and transgenic lines were grown in glasshouse on high porosity potting mix (52 Mix, Conard Fafard Inc., MA USA) supplied with $16 \mathrm{~h}$ day/8 $\mathrm{h}$ night photoperiod and $23^{\circ} \mathrm{C}$ day $/ 18^{\circ} \mathrm{C}$ night temperature conditions. Mature green (MG) fruits were characterized after 35-38 day post anthesis (DPA) and were denoted by green fully sized grown fruit. The Breaker (BR) stage was approximately 42 DPA and it was characterized by ripening-associated color shifting from green to yellow. Fruit of subsequent ripening stages were defined in "days post-breaker", like the ripening stage of four and eight days after breaker (BR4 and BR8), respectively. The fruits of WT, $556 \mathrm{HO}$, and $579 \mathrm{HO}$ genotypes were harvested at two stages of fruit ripening: $\mathrm{MG}$ and BR4 (unless otherwise stated) and kept at $-80^{\circ} \mathrm{C}$.

\section{RNA sequencing (RNASeq) Experiment and normalization of read counts}

Total RNA was extracted from pericarp tissues of tomato fruits as described previously ${ }^{[63]}$. Total RNA was pre-treated with RNase-free DNase (Promega) and cDNA was synthesized using Superscript II reverse transcriptase kit as previously described ${ }^{[45]}$. Quality control was made by using FastQC. Based on the result from FastQC, all bases in the reads in our samples have good quality (quality score> 30). Reads were aligned to Solanum lycopersicum L. genome using Tophat v 2.1.1 with default parameters ${ }^{[64]}$. Gene counts were calculated by HTSeq [65] using parameter "htseq-count -s no" (For stranded $=$ no, the data is not from a strand-specific assay, a read is considered overlapping with a feature regardless of whether it is mapped to the same or the opposite strand as the feature). The annotation information used in this step was downloaded from (https://solgenomics.net/organism/Solanum_lycopersicum /genome) Solanum lycopersicum L. Genomics Network. The number of mapped reads for a particular gene were scored as 'counts' and used for downstream analysis, including differential gene expression. The HTseq files for tomato at MG and BR4 old fruits from WT, 556HO, and $579 \mathrm{HO}$ were finally normalized in Reads per kilo base per million mapped reads (RPKM) reads ${ }^{[66-67]}$. Finally processed data file(s) included read counts and gene function annotation was used to analyze expression patterns of ATG8/ATG5 genes.

Total protein extraction, SDS-PAGE, and immunoblotting analyses

Pericarp tissue of tomato fruit was ground in liquid nitrogen and homogenized in sample buffer [68]. Extraction and fractionation of isolated total cellular proteins were performed on 10 and/or 15\% SDS-PAGE as previously described by ${ }^{[69]}$ and ${ }^{[70]}$, respectively. Lipidated (PE-conjugated) and unlipidated (PEunconjugated) forms of ATG8 were distinguished using 6M Urea-SDS-PAGE as previously described [71]. Protein electrophoretic separation was either followed by gel staining with Coomassie blue R-250 or blotting onto a nitrocellulose membrane $(0.1 \mu$, Schleicher \& Schull, Germany) in Towbin-buffer (192 mM Glycin, $25 \mathrm{mM}$ Tris/HCl, pH 8.3, $0.1 \%(\mathrm{w} / \mathrm{v})$ SDS, and $15 \%(\mathrm{v} / \mathrm{v})$ Methanol) using Bio-Rad Trans-Blot ${ }^{\circledR}$ Semi-Dry electrophoretic cell (Cat. number 170-3940) according to manufacturer's instructions. Polyclonal antibodies against ATG8, ATG5, and alkaline phosphataseconjugated 2ry anti-rabbit IgG (Agrisera) were used. Immunoblotting analyses were performed as described previously ${ }^{[72]}$.

\section{Statistical analysis}

A Microsoft Excel add-in statistical package XLSTAT (2014.3.05) was used for calculation and estimation of level of significance as revealed by TTEST, standard deviation $\left(\mathrm{S}_{\mathrm{DEV}}\right)$, standard error $\left(\mathrm{S}_{\mathrm{ERROR}}\right)$, and generating color schemes to investigate the fold change of differential gene(s) expression between WT and PAs genetically modified tomato cell lines.

\section{Results and Discussion}

\section{Identification of ATG8/ATG5 gene homologues}

Based on tomato genome sequence and gene ontology (GO) database, twenty-two putative autophagy-related genes were identified in Solanum lycopersicum L. (http://solgenomics.net/). However, in this study gene transcripts of nine putative autophagy genes of both ATG8 and ATG5 were represented in ripening fruit RNAseq transcriptomic data (listed in Table 1). Identified autophagy genes included seven ATG8 and two ATG5 transcripts that were detectable constantly in the three biological replicates of each ripening growth stage.

Diffrential gene expression of ATG8/ATG5 homologues during ripening of enhanced PAs tomato fruits

The SlE8-ySAMdc transgenic fruits accumulated up to 3fold higher spermidine (Spd) and spermine (Spm), exhibited several desirable attributes including higher carotenoids production, and extended shelf life ${ }^{[45]}$. Expression patterns of the autophagy genes (ATG8/ATG5) were examined during the ripening of SIE8-ySAMDC transgenic and their parental WT fruits to determine how enhanced PAs affected autophagy 
Table 1: Identification of ATG8/ATG5 gene homologues as revealed by carried out RNASeq experiment in this study and subsequent analysis of gene transcripts' read counts.

\begin{tabular}{lcc}
\hline \multicolumn{1}{c}{ Gene description } & Gene ID & Gene length \\
\hline Autophagy-related protein 5 (ATG5a) & Solyc06g043140.2 & 1094 \\
Autophagy-related protein 5 (ATG5b) & Solyc02g036380.2 & 14227 \\
Autophagy-related protein 8 (ATG8a) & Solyc02g080590.2 & 2724 \\
Autophagy-related protein 8 (ATG8b) & Solyc10g006270.2 & 2965 \\
Autophagy-related protein 8 (ATG8c) & Solyc07g064680.2 & 3005 \\
Autophagy-related protein 8 (ATG8d) & Solyc08g078820.2 & 5254 \\
Autophagy-related protein 8 (ATG8e) & Solyc03g031650.2 & 2938 \\
Autophagy-related protein 8 (ATG8f) & Solyc08g007400.2 & 1934 \\
Autophagy-related protein 8 (ATG8g) & Solyc01g068060.2 & 6086 \\
\hline
\end{tabular}

process in tomato fruits. The results have shown differential expression of ATG8/ATG5 genes during the ripening of WT as well as transgenic fruit $(556 \mathrm{HO}$ and $579 \mathrm{HO})$. Regarding the two identified ATG5 genes, the transcript levels of ATG5a was highly significant upregulated during $\mathrm{MG}$ stage of $579 \mathrm{HO}$ genotype compared to WT-MG fruit. However, the expression of ATG5a was significantly down-regulated in BR4 stage of the same genotype, $579 \mathrm{HO}$ (Figure 2, panel 1). Whereas the transcript levels of ATG5b was significantly down-regulated in MG stage of $556 \mathrm{HO}$ and recorded a highly significant up-regulation in BR4 stage (Figure 2, panel 2) compared to WT fruits. Taken together, these findings have shown that at least one ATG5 gene that was kept significantly up-regulated in each of shown growing stages of PAs-enhanced transgenic tomato cell lines (Figure 2, panels 1-2). The ATG5b exhibited the highest accumulation in BR4 stage of $556 \mathrm{HO}$ genotype. Hereby, these results suggested a pronounced role of PAs in alteration the gene expression profile of ATG5 gene(s) during fruit ripening.

Among the various ATG8 homologues, the seven ATG8 genes were expressed during fruit ripening. However, their expression based on steady state transcript accumulation varied significantly. Transcript levels of ATG8a, ATG8c, and ATG8f (Figure 2, panels 3, 5, and 8) were significantly down-regulated in both stages of $556 \mathrm{HO}$ and/or $579 \mathrm{HO}$ cell lines compared to WT corresponding stages. On the other hand, the expression of ATG8b, ATG8d, and ATG8e were significantly to highly significant up-regulated in MG and BR4 stages of $556 \mathrm{HO}$ and/or $579 \mathrm{HO}$ (Figure 2, panels 4, 6, and 7). The statistical analysis of gene transcripts' counts of ATGg (Solyc01g068060.2) has shown non-significance $(\mathrm{P}>0.05)$. Therefore, the results regarding this gene were not shown. Ripening of 556HO-MG stage significantly triggered the highest transcript accumulation levels of ATG8b, followed by ATG8d in 579HO-MG and ATG8e in both 556HO and $579 \mathrm{HO}$ genotypes compared to WT relevant stages.

These results collectively indicate that gene expression of autophagy genes of ATG8/ATG5 is altered during fruit ripening in PAs enriched genotypes as previously discussed [61,62]. However, additional experiments, especially monitoring and analysis of the transcriptomic profiles of autophagy genes responsible for other autophagosome targeting are needed. Also, localization studies focusing on changes in autophagosome formation (using in vivo labeling) during tomato fruit ripening are indispensable as future prospective to be further investigated.

Dissection of gene expression alteration of ATG8/ATG5 among enriched PAs genetically modified tomato cell lines

Since two independent homozygous genetically modified genotypes $(556 \mathrm{HO}$ and $579 \mathrm{HO}){ }^{[45]}$ were used in this study, it was prompting to generate a comparative analysis among $556 \mathrm{HO}$ and $579 \mathrm{HO}$ (referenced to WT read counts) analyzing their differentially expressed genes. Fold changes of ATG8/ATG5 related genes of two ripening stages $\mathrm{MG}$ and $\mathrm{BR} 4$, as revealed by RNASeq analysis, were calculated (Figure 3, panels AB). The data was analyzed by Excel-enabled XLSTAT Version 2014.4.06. Transcriptional analysis of expressed genes was represented as RPKM-fold changes compared to the relevant WT developmental stage by a color scale. Statistically decreased and increased transcript levels were shown in red and blue, respectively. Expression patterns (increasing/decreasing) of the ATG8/ATG5 genes in $556 \mathrm{HO}$ and $579 \mathrm{HO}$ at $\mathrm{MG}$ and BR4 stages exhibited a high degree of similarity (Figure 3, panels A-B, denoted by pale grey arrows). For example, ATG8a, ATG8e, and ATG8f have shown a pronounced degree of similarity between their fold changes of gene transcripts. These results reflected how conservative is the impact of engineered PAs in triggering an alteration $\mathrm{n}$ the gene expression profiles of ATG8/ATG5 genes. Notably, the fold change analysis of ATG5a has shown pronounced difference between the two transgenic lines (Figure 3, panels A-B, denoted by dark grey arrow). The latter finding could be attributed to the position in which the construct expressing yeast SAMdc gene 
$(y S A M d c)$ under SlE8 promoter was genetically incorporated ${ }^{[45]}$. Bioinformatics studies revealing the correlation coefficient and clustering analyses might be of interest as a future prospective for drawing a comprehensive understanding about the interplay between PAs and whole genes responsible for autophagy process.
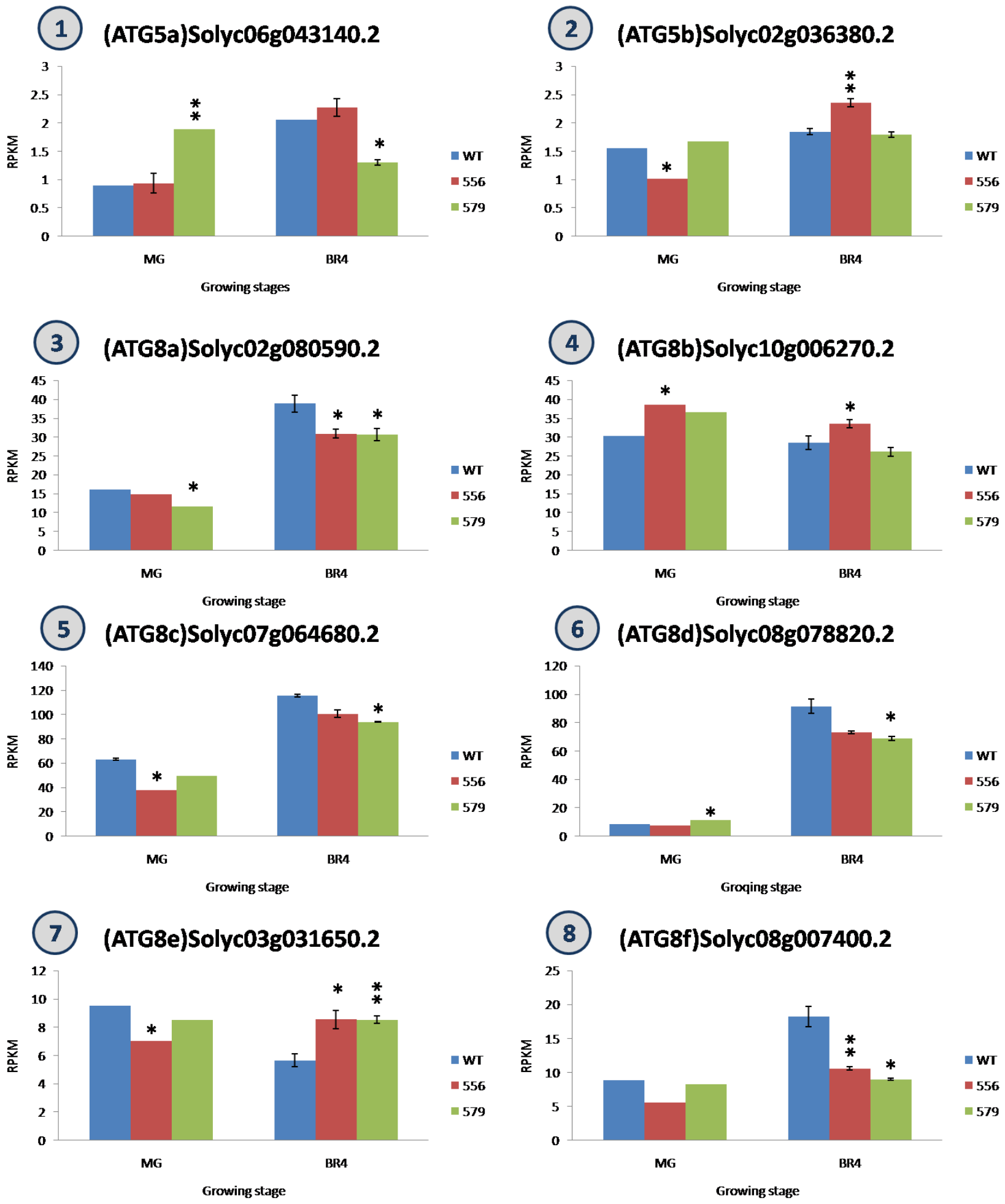

Fig. 2: Changes in the steady state transcript levels of ATG8 and ATG5 genes implicated in ATG8-PE conjugation system during autophagy process and ATG5-ATG12 conjugation system, respectively. RNASeq experiments were performed during ripening of WT (cv. Ohio 8245), independent transgenic tomato fruits enhanced in spermidine /spermine levels (556HO and 579HO). $\mathrm{X}$ axis represents the analyzed growing stages (MG and BR4) of tomato fruits. $\mathrm{Y}$ axis values refer to normalized read counts of differentially expressed genes in (RPKM) of three independent biological experiments. The data are shown as mean \pm S.E.M; $*, \mathrm{P}<0.05$, **, $\mathrm{P}<0.005$. 
A

\section{Fold change of differentially expressed genes in $556 \mathrm{HO}$}

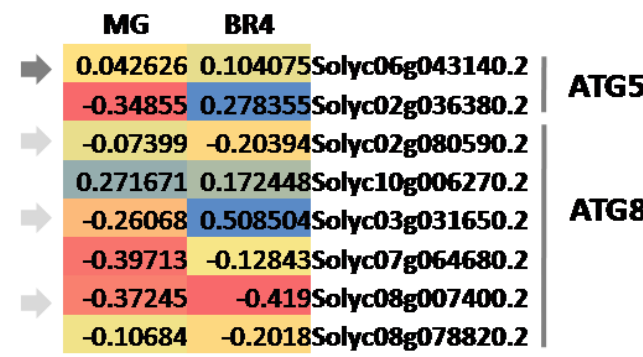

B

\section{Fold change of differentially expressed genes in $\mathbf{5 7 9 H O}$}

\begin{tabular}{|c|c|c|}
\hline MG & BR4 \\
\hline 1.10452 & -0.36597 Solyc06g043140.2 & ATG5 \\
\hline 0.073448 & -0.02693 Solyc02g036380.2 & AT \\
\hline-0.27391 & -0.21008 Solyc02g080590.2 & \\
\hline 0.210456 & -0.08587 Solyc10g006270.2 & \\
\hline-0.10888 & 0.506731 Solyc03g031650.2 & ATG8 \\
\hline-0.21617 & -0.18627 Solyc07g064680.2 & \\
\hline-0.06018 & -0.50656 Solyc08g007400.2 & \\
\hline 0.346686 & -0.24837 Solyc08g078820.2 &
\end{tabular}

Fig. 3: Fold changes of ATG8/ATG5 related genes of two ripening stages MG and BR4 as revealed by RNASeq analysis. Panel (A) and panel (B) showing analysis of fold changes of differentially expressed ATG8/ATG5 gene transcripts in PAs enriched homozygous cell lines $556 \mathrm{HO}$ and $579 \mathrm{HO}$, respectively, at two stages of ripening of tomato ripening fruits. The data was analyzed by Excel-enabled XLSTAT Version 2014.4.06. Transcriptional analysis of expressed genes was represented as RPKM-fold changes compared to the relevant WT developmental stage by a color scale. Red and blue shows statistically decreased and increased transcript levels, respectively. Similar and different gene expression gene profile(s) between $556 \mathrm{Ho}$ and $579 \mathrm{Ho}$ transgenic cell lines were depicted as pale and dark grey arrows.

Immunoblotting analysis of ATG8/ATG5 levels and their autophagic triggered conjugates

To manifest if the generated genotypes (ySAMdc-high spermidine/spermine homozypous tomato cell lines; 556 and 579) affected the accumulation of the ATG5 protein and its relative conjugates, immunoblot analysis was performed on total cellular protein extracts from tomato pericarp tissues through four stages of fruit ripening. These stages were mature green (MG), breaker (BR), breaker+4 days (BR4), and breaker+8days (BR8). Immunoblot analysis was performed using antibody against ATG5 protein. During the mature green stage of fruit ripening (Figure 4, panel A) minor proteins at approximately $40-\mathrm{kDA}$ and $50-\mathrm{kDa}$ were close to apparent mass of predicted free ATG5 (38.479 kDa) and conjugated ATG5-ATG12 (49.033 kDa), respectively. Notably, an abundant $50-\mathrm{kDa}$ protein (hereinafter referred to as ATG5-ATG12 conjugate) was detected in PAs enhanced 579 cell line (Figure 4, panel A, lane 3). Likely, conjugated ATG5-ATG12 was pronouncedly detected at breaker stage in PAs accumulating 556 tomato cell line (Figure 4, panel B, lane 2). ATg5ATG12 conjugate were equally detected more abundant than free ATG5 in BR4 and BR8 ripening stages either in WT or PAs genetically modified lines 556 and 579 (Figure 4, panels C and D). Newly identified ATg5related conjugates were slightly more abundant at BR8 stage in both PAs enhanced lines (Figure 4, panels D, lanes 2-3, denoted by black arrow head). Therefore, it is most likely speculated PAs enhancement has triggered abundant conjugation of ATG5-ATG12 in earlier stages of fruit ripening (specifically at MG and BR). The same findings of triggering abundant ATG5-ATG12 conjugate under unfavorable $\mathrm{C}$-starvation conditions were previously investigated ${ }^{[32]}$.
To access the possibility that fluctuation of ATG5 expression affects ATG8 conjugation pathways in tomato as it does in Arabidopsis ${ }^{\text {[32] }}$ and in yeast ${ }^{[73]}$, ATG8 protein and it relevant conjugate with phosphatidyl ethanolamine (ATG8-PE) was assayed during previously described fruit ripening stages. ATG8 protein isoforms (Figure 5, panels A-D, denoted by star) were identified in near equal amounts in all examined four stages. ATG8-PE adduct was identified in 556 PAs enhanced mutant at MG ripening stage (albeit weak) in urea-supplemented SDS-PAGE (Figure 5, panel $\mathrm{A}$, middle part, lane 2) at approximately $10-\mathrm{kDa}$ based on their faster migration ${ }^{[32]}$. ATG8-PE adduct was previously identified at the same molecular weight as shown by ${ }^{[74]}$ and ${ }^{[75]}$ during $\mathrm{N}$ - and C-starvation, respectively. Weak identification of ATG8-PE conjugate might be attributed for detection limits of ALPconjugated 2ry anti-rabbit IgG (Agrisera). On the same context, during the BR8 stage several newly ATG8related conjugates have become predominantly identified (ranging from approximately $40-\mathrm{kDa}$ to $75-\mathrm{kDa}$ and denoted by black arrow head) specifically in PAs engineered mutants (Figure 5, panel D, middle part, lanes 2-3).

To conclude, the ripened fruit maintained high levels of ATG8 to actively participate in the autophagy process during fruit ripening. There is an ongoing association between autophagy activation and longevity ${ }^{[75]}$. Recent developing evidence has suggested that reduced autophagic activity may play a critical role in the aging process. Aged cells are characterized by a progressive accumulation of non-functional cellular components due to oxidative damage and a failure in recycling level and housekeeping mechanisms. The cytoprotective function of autophagy allows the cells to survive under conditions 
of nutrient deprivation by the self-cannibalization of cellular organelles ${ }^{[5]}$. However, the role of this process in maintaining fruit quality by delaying senescence/aging process remains and needs to be further investigated. Putting into consideration the differential expression (on transcriptional level) and conjugation behavior of ATG5 and ATG8 in PAs enhanced genotypes with ATG12 and PE (on translational level), respectively, it might be speculated that the latter two proteins may play a significant role in dissecting and deciphering the mechanistic pathway of autophagy process.

\section{Acknowledgment}

All of gratitude and appreciation to respected Prof. Dr. Avtar K. Handa for his keen support and kind efforts throughout performing the experimental procedures of the present study. Thanks are extended to Dr. Marwa A. Fakhr for her kind support and keen help during the preparation this manuscript and for all lab members of Prof. Dr. Avtar K. Handa, especially Tatsiana U. Datsenka for her kind help during this study.

B
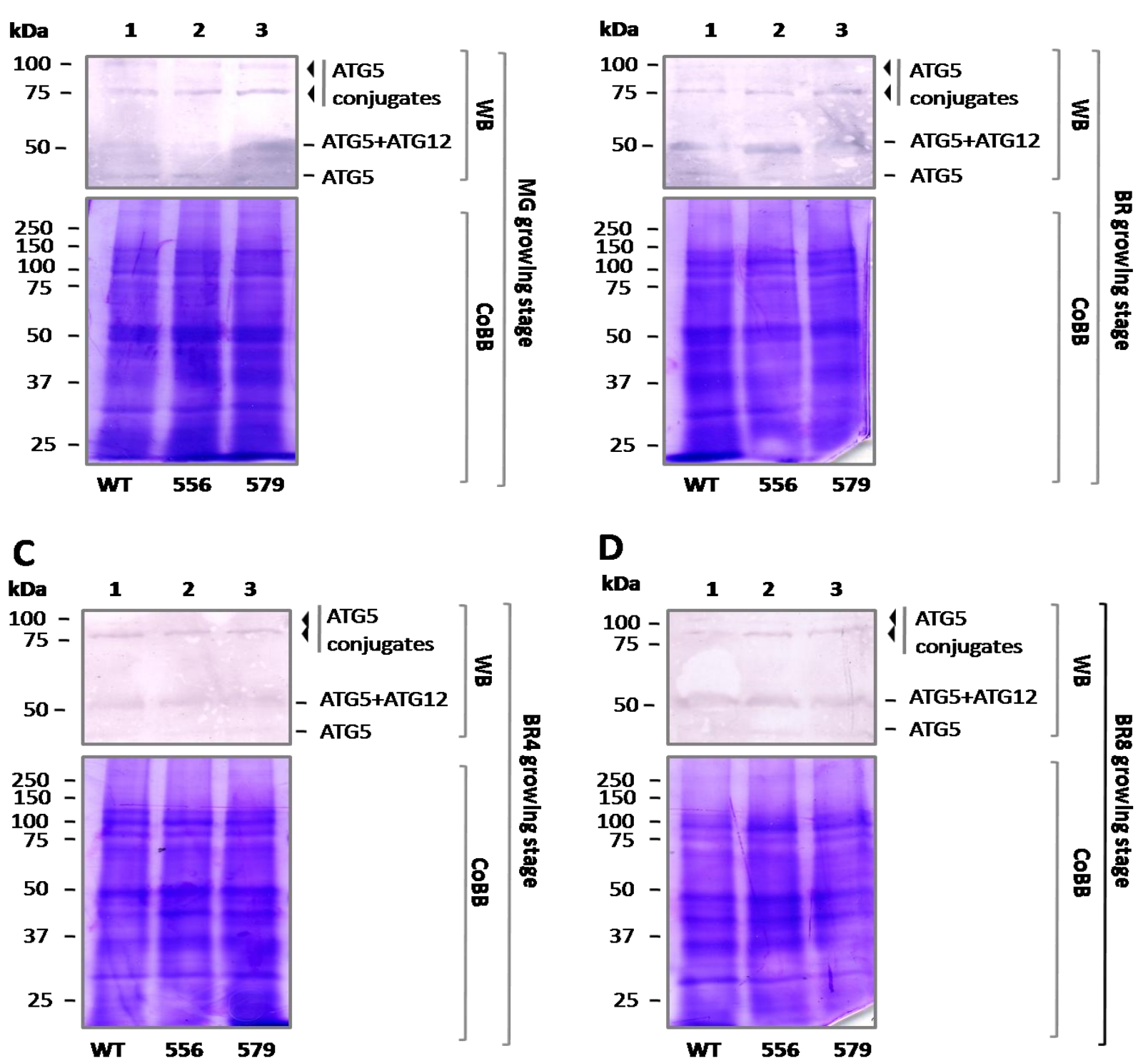

Fig. 4: Immunoblot analysis of autophagy protein ATG5 and related conjugates in polyamines enhanced genetic modified tomato cell lines at MG, BR, 4 and 8 days after breaker (BR4 and BR8, respectively). A, B, C, and D, SDS-PAGE analysis of extracted total cellular proteins (lower panels) stained by COBB from an equal amount of liquid $\mathrm{N}_{2}$ ground pericarp tissue. Unified equal loaded amounts were manifested by COBB staining. Gels were subjected to immunoblot (western blot, WB) with antibody against autophagy-related protein 5 (ATG5) either without (Upper panels) or with (middle panels) denaturing 6M urea. Total cellular protein extracts were isolated from WT (lane 1), 556HO and $579 \mathrm{HO}$ (lanes 2-3 respectively) were demonstrated. Free ATG5 and presumed ATG5ATG12 conjugate were detected at 40 and $50 \mathrm{kDa}$, respectively. Black arrowheads denoted the presumed other detected ATG5-related conjugates demonstrated in this study. The numbers shown on the left-handed side of the figures indicate molecular weight standards in $\mathrm{kDa}$. 
$\mathbf{A}$

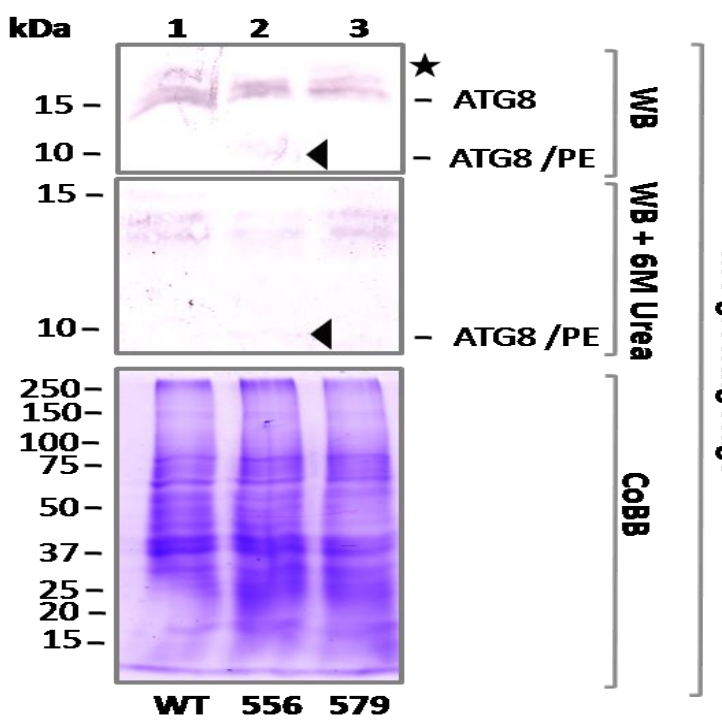

B

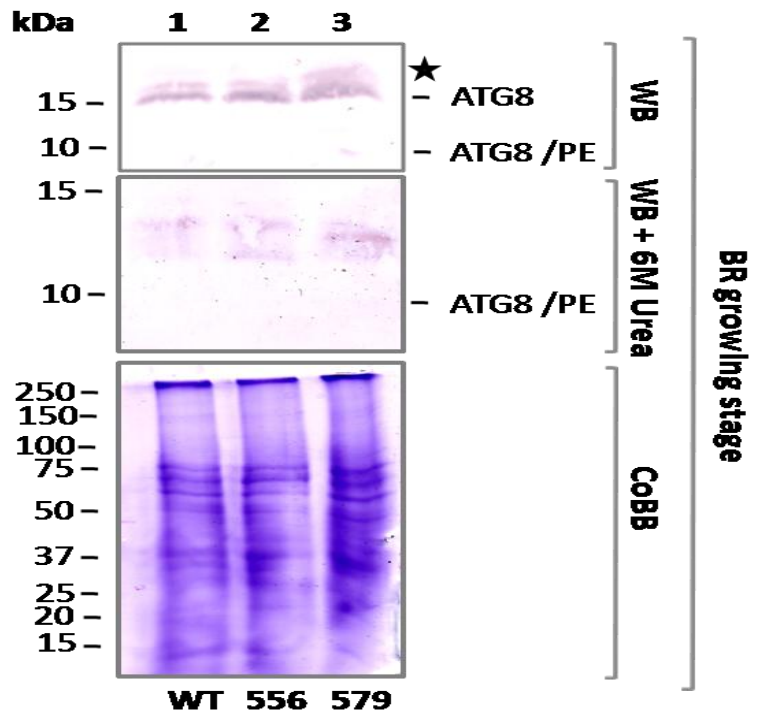

D

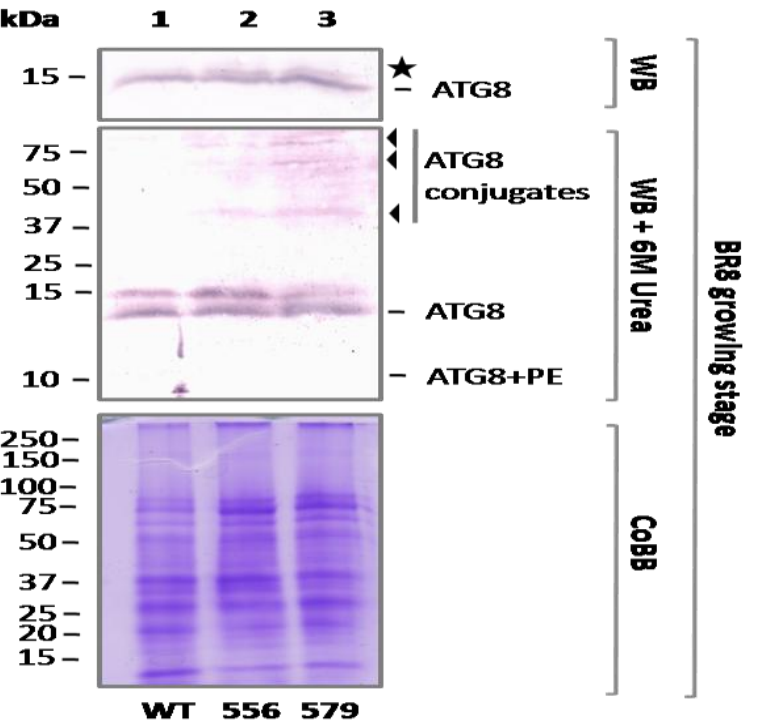

Fig. 5: Immunoblot analysis of autophagy protein ATG8 and related conjugates in polyamines enhanced genetic modified tomato cell lines at MG, BR, 4 and 8 days after breaker (BR4 and BR8, respectively). A, B, C, and D, SDS-PAGE analysis of extracted total cellular proteins (lower panels) stained by Coomassie Brilliant Blue G250 (COBB) from an equal amount of liquid $\mathrm{N}_{2}$ ground pericarp tissue. Unified equal loaded amounts were manifested by COBB staining. Gels were subjected to immunoblot (western blot, WB) with antibody against autophagy-related protein 8 (ATG8) either without (Upper panels) or with (middle panels) denaturing 6M urea. Total cellular protein extracts were isolated from WT (lane 1), 556HO and $579 \mathrm{HO}$ (lanes 2-3 respectively) were demonstrated. Black arrowheads denoted the presumed ATG8-PE (Phosphatidyl ethanolamine) adducts or other detected ATG8-related conjugates demonstrated in this study. Proposed ATG8 isoforms were denoted by asterisk in black bold print. The numbers shown on the left-handed side of the figures indicate molecular weight standards in $\mathrm{kDa}$.

\section{References}

1) Vierstra, R. D. (1996). Proteolysis in plants: mechanisms and functions. Plant molecular biology, 32(1-2):275-302.

2) Smalle, J. and Vierstra, R. D. (2004). The ubiquitin $26 \mathrm{~S}$ proteasome proteolytic pathway. Annual review of plant biology, 55:555-590.

3) Rabinowitz, J. D. and White, E. (2010). Autophagy and metabolism. Science, 330(6009): 1344-1348.
4) Klionsky, D. J. (2007). Autophagy: from phenomenology to molecular understanding in less than a decade. Nature reviews Molecular cell biology, 8(11):931-937.

5) Liu, Y. and Bassham, D. C. (2012). Autophagy: pathways for self-eating in plant cells. Annual review of plant biology, 63:215-237.

6) Bassham, D. C. (2007). Plant autophagy-more than a starvation response. Current opinion in plant biology, 10(6):587-593. 
7) Guiboileau, A., Sormani, R., Meyer, C. and Masclaux-Daubresse, C. (2010). Senescence and death of plant organs: nutrient recycling and developmental regulation. Comptes rendus biologies, 333(4):382-391.

8) Backues, S. K. and Klionsky, D. J. (2011). Autophagy gets in on the regulatory act. Journal of molecular cell biology, 3(2):76-77.

9) Lenz, H. D., Haller, E., Melzer, E., Kober, K., Wurster, K., Stahl, M. and Molina, A. (2011). Autophagy differentially controls plant basal immunity to biotrophic and necrotrophic pathogens. The Plant Journal, 66(5):818-830.

10) Grisolia, S., Knecht, E. R. W. I. N., HernándezYago, J. O. S. E., and Wallace, R. (1980). Turnover and degradation of mitochondria and their proteins. In Protein Degradation in Health and Disease (pp. 167-188). Excerpta Medica Amsterdam.

11) Youle, R. J. and Narendra, D. P. (2011). Mechanisms of mitophagy. Nature reviews Molecular cell biology, 12(1):9-14.

12) Avin-Wittenberg, T., Bajdzienko, K., Wittenberg, G., Alseekh, S., Tohge, T., Bock, R. and Fernie, A. R. (2015). Global Analysis of the Role of Autophagy in Cellular Metabolism and Energy Homeostasis in Arabidopsis Seedlings under Carbon Starvation. The Plant Cell Online, 27(2): 306-322.

13) Guo, J. Y., Chen, H. Y., Mathew, R., Fan, J., Strohecker, A. M., Karsli-Uzunbas, G. and Coller, H. A. (2011). Activated Ras requires autophagy to maintain oxidative metabolism and tumorigenesis. Genes \& development, 25(5):460470.

14) Liu, Y., Schiff, M., Czymmek, K., Tallóczy, Z., Levine, B. and Dinesh-Kumar, S. P. (2005). Autophagy regulates programmed cell death during the plant innate immune response. Cell, 121(4): 567-577.

15) Patel, S. and Dinesh-Kumar, S. P. (2008). Arabidopsis ATG6 is required to limit the pathogenassociated cell death response. Autophagy, 4(1):20 27.

16) Yoshimoto, K., Jikumaru, Y., Kamiya, Y., Kusano, M., Consonni, C., Panstruga, R. and Shirasu, K. (2009). Autophagy negatively regulates cell death by controlling NPR1-dependent salicylic acid signaling during senescence and the innate immune response in Arabidopsis. The Plant Cell, 21(9):2914- 2927.

17) Wang, S. and Blumwald, E. (2014). Stress-induced chloroplast degradation in Arabidopsis is regulated via a process independent of autophagy and senescence-associated vacuoles. The Plant Cell, 26(12):4875-4888.

18) Levine, B. and Kroemer, G. (2008). Autophagy in the pathogenesis of disease. Cell, 132(1):27-42.

19) Mizushima, N., Levine, B., Cuervo, A. M. and Klionsky, D. J. (2008). Autophagy fights disease through cellular self-digestion. Nature, 451(7182): 1069-1075.

20) Farré, J. C., Krick, R., Subramani, S. and Thumm, M. (2009). Turnover of organelles by autophagy in yeast. Current opinion in cell biology, 21(4):522-530.

21) Behrends, C., Sowa, M. E, Gygi, S. P. and Harper, J. W. (2010). Network organization of the human autophagy system. Nature, 46(6):68-76.

22) Johansen, T. and Lamark, T. (2011). Selective autophagy mediated by autophagic adapter proteins. Autophagy, 7(3):279-296.

23) Vilchez, D., Saez, I. and Dillin, A. (2014). The role of protein clearance mechanisms in organismal ageing and age-related diseases. Nature communications, 5:5659.

24) Liu, Y., Xiong, Y. and Bassham, D. C. (2009). Autophagy is required for tolerance of drought and salt stress in plants. Autophagy, 5(7):954-963.

25) Mizushima, N. and Komatsu, M. (2011). Autophagy: renovation of cells and tissues. Cell, 147(4):728-741.

26) Reggiori, F. and Klionsky, D. J. (2013). Autophagic processes in yeast: mechanism, machinery and regulation. Genetics, 194(2):341-361.

27) Xie, Z. and Klionsky, D. J. (2007). Autophagosome formation: core machinery and adaptations. Nature cell biology, 9(10):1102-1109.

28) Avin-Wittenberg, T., Honig, A. and Galili, G. (2011). Variations on a theme: plant autophagy in comparison to yeast and mammals. Protoplasma, 249(2):285-299.

29) Hanada, T., Noda, N. N., Satomi, Y., Ichimura, Y., Fujioka, Y., Takao, T. and Ohsumi, Y. (2007). The Atg12-Atg5 conjugate has a novel E3-like activity for protein lipidation in autophagy. Journal of Biological Chemistry, 282(52):37298-37302.

30) Noda, N. N., Fujioka, Y., Hanada, T., Ohsumi, Y. and Inagaki, F. (2013). Structure of the Atg12-Atg5 conjugate reveals a platform for stimulating Atg8-PE conjugation. EMBO Reports, 14(2):206-11.

31) Gasch, A. P., Spellman, P. T., Kao, C. M., Carmel-Harel, O., Eisen, M. B., Storz, G. and Brown, P. O. (2000). Genomic expression programs in the response of yeast cells to environmental changes. Molecular biology of the cell, 11(12): 4241-4257.

32) Thompson, A. R. and Vierstra, R. D. (2005). Autophagic recycling: lessons from yeast help define the process in plants. Current opinion in plant biology, 8(2): 165-173.

33) Brauer, M. J., Huttenhower, C., Airoldi, E. M., Rosenstein, R., Matese, J. C., Gresham, D. and Botstein, D. (2008). Coordination of growth rate, cell cycle, stress response, and metabolic activity in yeast. Molecular biology of the cell, 19(1):352-367.

34) Ryter, S. W., Cloonan, S. M. and Choi, A. M. (2013). Autophagy: a critical regulator of cellular metabolism and homeostasis. Molecules and cells, 36(1):7-16. 
35) Ohsumi, Y. (2014). Historical landmarks of autophagy research. Cell research, 24(1):9-23.

36) Chung, T., Suttangkakul, A. and Vierstra, R. D. (2009). The ATG autophagic conjugation system in maize: ATG transcripts and abundance of the ATG8-lipid adduct are regulated by development and nutrient availability. Plant Physiology, 149(1):220-234.

37) Li, F., Chung, T., Pennington, J. G., Federico, M. L., Kaeppler, H. F., Kaeppler, S. M. and Vierstra, R. D. (2015). Autophagic recycling plays a central role in maize nitrogen remobilization. The Plant Cell, 27(5):1389-1408.

38) Xia, K., Liu, T., Ouyang, J., Wang, R., Fan, T. and Zhang, M. (2011). Genome-wide identification, classification, and expression analysis of autophagy-associated gene homologues in rice (Oryza sativa L.). DNA research, 18(5):363-377.

39) Kurusu, T., Koyano, T., Hanamata, S., Kubo, T., Noguchi, Y., Yagi, C. and Kitahata, N. (2014). OsATG7 is required for autophagy-dependent lipid metabolism in rice postmeiotic anther development. Autophagy, 10(5):878-888.

40) Kuzuoglu-Ozturk, D., Yalcinkaya, O. C., Akpinar, B. A., Mitou, G., Korkmaz, G., Gozuacik, D. and Budak, H. (2012). Autophagyrelated gene, TdAtg8, in wild emmer wheat plays a role in drought and osmotic stress response. Planta, 236(4):1081-1092.

41) Zhou, J., Wang, J., Yu, J. Q. and Chen, Z. (2014). Role and regulation of autophagy in heat stress responses of tomato plants. Front Plant Sci., 30(5): 174 .

42) Adams-Phillips, L., Barry, C. and Giovannoni, J. (2004). Signal transduction systems regulating fruit ripening. Trends in plant science, 9(7):331-338.

43) Srivastava, A. and Handa, A. K. (2005). Hormonal regulation of tomato fruit development: a molecular perspective. Journal of Plant Growth Regulation, 24(2):67-82.

44) Barry, C. S. and Giovannoni, J. J. (2007). Ethylene and fruit ripening. Journal of Plant Growth Regulation, 26(2):143-159.

45) Mehta, R. A., Cassol, T., Li, N., Ali, N., Handa, A. K. and Mattoo, A. K. (2002). Engineered polyamine accumulation in tomato enhances phytonutrient content, juice quality, and vine life. Nature biotechnology, 20(6):613-618.

46) Eisenberg, T., Knauer, H., Schauer, A., Büttner, S., Ruckenstuhl, C., Carmona-Gutierrez, D. and Fussi, H. (2009). Induction of autophagy by spermidine promotes longevity. Nature cell biology, 11(11):1305-1314.

47) Nambeesan, S., Datsenka, T., Ferruzzi, M. G., Malladi, A., Mattoo, A. K. and Handa, A. K. (2010). Overexpression of yeast spermidine synthase impacts ripening, senescence and decay symptoms in tomato. The plant journal, 63(5):836847.
48) Veress, I., Haghighi, S., Pulkka, A. and Pajunen, A. (2000). Changes in gene expression in response to polyamine depletion indicates selective stabilization of mRNAs. Biochemical Journal, 346(1):185-191.

49) Kasukabe, Y., He, L., Nada, K., Misawa, S., Ihara, I. and Tachibana, S. (2004). Overexpression of spermidine synthase enhances tolerance to multiple environmental stresses and up-regulates the expression of various stress-regulated genes in transgenic Arabidopsis thaliana.Plant and Cell Physiology, 45(6):712-722.

50) Yoshida, M., Kashiwagi, K., Shigemasa, A., Taniguchi, S., Yamamoto, K., Makinoshima, H. and Igarashi, K. (2004). A unifying model for the role of polyamines in bacterial cell growth, the polyamine modulon. Journal of Biological Chemistry, 279(44):46008-46013.

51) Alcázar, R., Marco, F., Cuevas, J. C., Patron, M., Ferrando, A., Carrasco, P. and Altabella, T. (2006). Involvement of polyamines in plant response to abiotic stress. Biotechnology letters, 28(23):18671876.

52) Igarashi, K. and Kashiwagi, K. (2006). Polyamine modulon in Escherichia coli: genes involved in the stimulation of cell growth by polyamines. Journal of biochemistry, 139(1):11-16.

53) Mattoo, A. K. and Handa, A. K. (2008). Higher polyamines restore and enhance metabolic memory in ripening fruit. Plant Science, 174(4):386-393.

54) Nambeesan, S., Mattoo, A. K. and Handa, A. K. (2008). Polyamines and regulation of ripening and senescence. Postharvest Biology and Technology of Fruits, Vegetables and Flowers, IA:319-340.

55) Handa, A. K. and Mattoo, A. K. (2010). Differential and functional interactions emphasize the multiple roles of polyamines in plants. Plant Physiology and Biochemistry, 48(7):540-546.

56) Galston, A. W. and Kaur-Sawhney, R. (1995). Polyamines as endogenous growth regulators. In: Plant Hormones: Physiology, Biochemistry, and Molecular Biology (ed., P.J. Davies), Kluwer Academic Publishers, Norwell, MA, pp. 158-178.

57) Cassol, T. and Mattoo, A. K. (2003). Do polyamines and ethylene interact to regulate plant growth, development and senescence?. Molecular Insight in Plant Biology. Science Publisher, Enfield, NH, 121-132.

58) Casero, R. A. and Marton, L. J. (2007). Targeting polyamine metabolism and function in cancer and other hyperproliferative diseases. Nature reviews Drug discovery, 6(5):373-390.

59) Thomas, T. and Thomas, T. J. (2001). Polyamines in cell growth and cell death: molecular mechanisms and therapeutic applications. Cellular and Molecular Life Sciences CMLS, 58(2):244-258.

60) Serafini-Fracassini, D., Del Duca, S., Monti, F., Poli, F., Sacchetti, G., Bregoli, A. M. and Della Mea, M. (2002). rTansglutaminase activity during 
senescence and programmed cell death in the corolla of tobacco (Nicotiana tabacum) flowers. Cell death and differentiation, 9(3):309321.

61) Kausch, K. D., Sobolev, A. P., Goyal, R. K., Fatima, T., Laila-Beevi, R., Saftner, R. A. and Mattoo, A. K. (2012). Methyl jasmonate deficiency alters cellular metabolome, including the aminome of tomato (Solanum lycopersicum L.) fruit. Amino acids, 42(2-3):843-856.

62) Fatima, T., Sobolev, A. P., Teasdale, J. R., Kramer, M., Bunce, J., Handa, A. K. and Mattoo, A. K. (2016). Fruit metabolite networks in engineered and non-engineered tomato genotypes reveal fluidity in a hormone and agroecosystem specific manner. Metabolomics, 12(6):1-15.

63) Eggermont, K., Goderis, I. J. and Broekaert, W. F. (1996). High-throughput RNA extraction from plant samples based on homogenisation by reciprocal shaking in the presence of a mixture of sand and glass beads. Plant Molecular Biology Reporter, 14(3):273-279.

64) Trapnell, C., Pachter, L. and Salzberg, S. L. (2009). TopHat: discovering splice junctions with RNA-Seq. Bioinformatics, 25(9):1105-11. doi: 10.1093/bioinformatics/btp120.

65) Anders, S., Pyl, P. T. and Huber, W. (2014). HTSeq-a Python framework to work with highthroughput sequencing data. Bioinformatics, btu638.

66) Mortazavi, A., Williams, B. A., McCue, K., Schaeffer, L. and Wold, B. (2008). Mapping and quantifying mammalian transcriptomes by RNASeq. Nature methods, 5(7):621-628.

67) Dillies, M. A., Rau, A., Aubert, J., HennequetAntier, C., Jeanmougin, M., Servant, N. and Guernec, G. (2013). A comprehensive evaluation of normalization methods for Illumina highthroughput RNA sequencing data analysis. Briefings in bioinformatics, 14(6):671-683.

68) Laemmli, U. K. (1970). Cleavage of structural proteins during the assembly of the head of bacteriophage T4. Nature 227:680-685.

69) Biggs, M. S., Harriman, R. W. and Handa, A. K. (1986). Changes in gene expression during tomato fruit ripening. Plant physiology, 81(2):395-403.

70) Mehta, R. A., Fawcett, T. W., Porath, D. and Mattoo, A. K. (1992). Oxidative stress causes rapid membrane translocation and in vivo degradation of ribulose-1, 5-bisphosphate carboxylase/oxygenase. Journal of Biological Chemistry, 267(4):2810-2816.

71) Kirisako, T., Ichimura, Y., Okada, H., Kabeya, Y., Mizushima, N., Yoshimori, T., Ohsumi, M., Takao, T., Noda, T. and Ohsumi Y. (2000). The reversible modification regulates the membranebinding state of Apg8/Aut7 essential for autophagy and the cytoplasm to vacuole targeting pathway. $J$. Cell Biol. 151:263-276.

72) Kausch, K. D. and Handa, A. K. (1997). Molecular cloning of a ripening specific lipoxygenase and its expression during wild-type and mutant tomato fruit development. Plant Physiol 113:1041-1050.

73) Suzuki, K., Kirisako, T., Kamada, Y., Mizushima, N., Noda, T. and Ohsumi Y. (2001). The preautophagosomal structure organized by concerted functions of APG genes is essential for autophagosome formation. EMBO J, 20:5971-5981.

74) Yoshimoto, K., Hanaoka, H., Sato, S., Kato, T., Tabata, S., Noda, T. and Ohsumi, Y. (2004). Processing of ATG8s, ubiquitin-like proteins, and their deconjugation by ATG4s are essential for plant autophagy. Plant Cell, 16:2967-2983.

75) Madeo, F., Tavernarakis, N. and Kroemer, G. (2010). Can autophagy promote longevity? Nature cell biology, 12(9):842-846. 